\title{
A multi-mode digital holographic microscope
}

\author{
Cite as: Rev. Sci. Instrum. 90, 023705 (2019); doi: 10.1063/1.5066556 \\ Submitted: 15 October 2018 - Accepted: 31 January 2019 • \\ Published Online: 25 February 2019
}

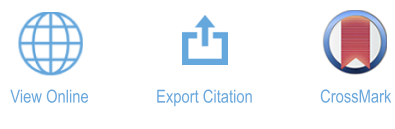

James L. Flewellen, ${ }^{1,2,3}$ (D) Irwin M. Zaid, ${ }^{3}$ and Richard M. Berry

\begin{abstract}
AFFILIATIONS
${ }^{1}$ Immune Receptor Activation Laboratory, The Francis Crick Institute, London NW1 1AT, United Kingdom

${ }^{2}$ Division of Immunology and Inflammation, Department of Medicine, Imperial College London, London SW7 2A2, United Kingdom

${ }^{3}$ Department of Physics, Clarendon Laboratory, University of Oxford, Parks Road, Oxford OX1 3PU, United Kingdom
\end{abstract}

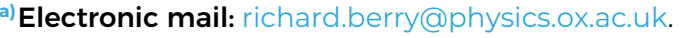

\begin{abstract}
We present a transmission-mode digital holographic microscope that can switch easily between three different imaging modes: inline, dark field off-axis, and bright field off-axis. Our instrument can be used: to track through time in three dimensions microscopic dielectric objects, such as motile micro-organisms; localize brightly scattering nanoparticles, which cannot be seen under conventional bright field illumination; and recover topographic information and measure the refractive index and dry mass of samples via quantitative phase recovery. Holograms are captured on a digital camera capable of high-speed video recording of up to 2000 frames per second. The inline mode of operation can be easily configurable to a large range of magnifications. We demonstrate the efficacy of the inline mode in tracking motile bacteria in three dimensions in a $160 \mu \mathrm{m} \times 160 \mu \mathrm{m} \times 100 \mu \mathrm{m}$ volume at $45 \times$ magnification. Through the use of a novel physical mask in a conjugate Fourier plane in the imaging path, we use our microscope for high magnification, dark field off-axis holography, demonstrated by localizing $100 \mathrm{~nm}$ gold nanoparticles at 225x magnification up to at least $16 \mu \mathrm{m}$ from the imaging plane. Finally, the bright field off-axis mode facilitates quantitative phase microscopy, which we employ to measure the refractive index of a standard resolution test target and to measure the dry mass of human erythrocytes.
\end{abstract}

(C) 2019 Author(s). All article content, except where otherwise noted, is licensed under a Creative Commons Attribution (CC BY) license (http://creativecommons.org/licenses/by/4.0/). https://doi.org/10.1063/1.5066556

\section{INTRODUCTION}

Digital holographic microscopy is a promising technique for high-speed, quantitative studies of the three-dimensional (3D) swimming behavior of micro-organisms, the 3D profiling of transient and steady-state flows both in biological contexts and in microfluidic devices, and topographical studies of cells and other microscopic objects. Holography stores three-dimensional information in a 2D image by encoding both the amplitude and phase of light incident on the recording medium. ${ }^{\top}$ Inclusion of a lens, such as a microscope objective, in the imaging path enables the 3D localization of micro-scale dielectric objects, such as micro-organisms, cells, or polystyrene microspheres, along with brightly scattering gold nanoparticles. ${ }^{2-5}$ Surface profiles and refractive indices of these objects can also be measured. ${ }^{6}$ Recent developments in both digital camera technology and high-speed computing ${ }^{7}$ mean digital holograms can be recorded at high frame rates, processed efficiently by computer hardware and the resulting 3D data analyzed in a numerical environment.

A hologram is formed by the interference of coherent light scattered off an object with a mutually coherent reference beam. The amplitude and phase information of the object beam is recovered through a numerical "re-illumination" of the hologram by a digital copy of the reference beam. This leads to a 3D "reconstruction" of the original object field, from which particles in the sample can be localized in three dimensions in each frame of a video recording. ${ }^{8,9}$ One issue in holographic reconstruction is that the procedure results in both real and virtual images of the sample being generated. These images must be isolated from one another, or their presence compensated for, during analysis. ${ }^{10}$

There are two geometrical approaches, in general, to recording a hologram: "inline" imaging, whereby the object 
and reference beams are aligned, and "off-axis" imaging, where the two beams interfere at an angle ${ }^{11}$ (see Fig. 1). The effect of the angle in off-axis holography is to separate the spatial frequencies of the real and virtual images from one another and from the unscattered illumination beam. Spatial frequency spectrum filtering can then be applied to the

Inline

a recording

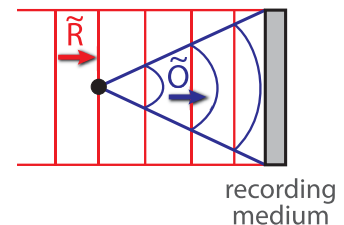

b reconstruction

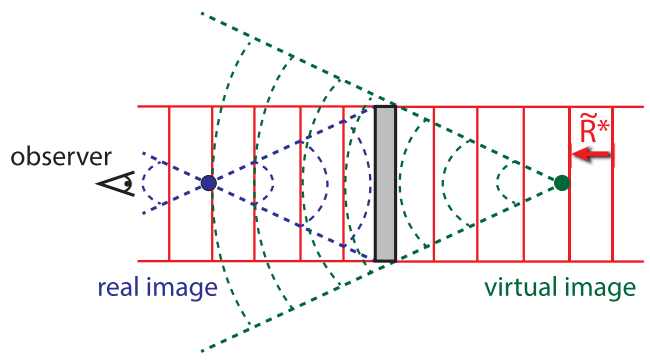

Off-axis

c recording
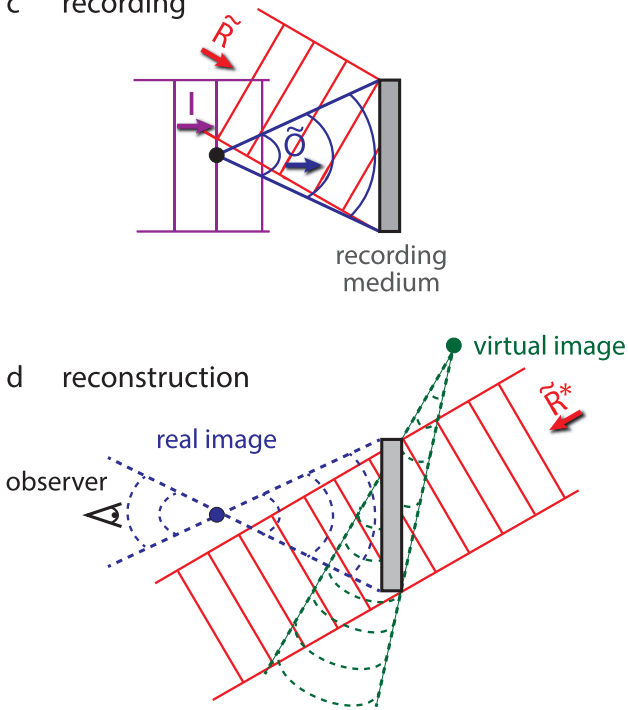

FIG. 1. Recording and reconstructing digital holograms. Inline holograms (a) result from interference between light scattered off the object (Õ) and a collinear reference beam $(\tilde{R})$. The reconstruction process $(b)$ is akin to illuminating the recording medium from the reverse side with a conjugate reference beam $\left(\tilde{R}^{*}\right)$ and overlays the real and virtual images from the point of view of the observer. In off-axis recording (c), the reference beam is introduced at an angle. The illumination beam (I) is typically blocked or removed digitally in post-processing. Off-axis reconstruction (d) results in a separation of real and virtual images. off-axis hologram to isolate the real image (see Fig. 4), allowing for reconstruction of the complex electromagnetic field of the object beam. ${ }^{12}$ This method necessarily generates a lower spatial resolution reconstruction as only a portion of the available frequency space is utilized; however, it does result in phase information acquired at the maximum time resolution. By contrast, in inline holography, the real and virtual images may only be separated from one another by recording multiple images per time point, thus reducing the temporal resolution of the technique. ${ }^{13-15}$ However, through using a suitably transparent sample and ensuring the distance between the illumination source and sample is several thousand times the wavelength, the signal from the virtual image becomes negligible. ${ }^{16}$ These criteria are easy to meet with aqueous, biological samples.

The application of digital holography to microscopy demonstrates the promise of this technique for studying the three-dimensional swimming behavior of micro-organisms either by observing the organisms directly or by particle image velocimetry (PIV) studies of the fluid around the swimmer. ${ }^{17-19}$ In the field of micro-organism motility, digital holographic microscopy has been used to study algae, ${ }^{20}$ copepods, ${ }^{21,22}$ dinoflagellates, ${ }^{23}$ and sperm. ${ }^{24}$ Unlike many other forms of 3D imaging, the temporal resolution of digital holographic microscopy is limited only by the frame rate of the camera. The technique does not rely on fluorescent particles and is suited to the study of many micro-scale objects moving dynamically in a single field of view. In the specific context of bacterial motility, ${ }^{25-27}$ this technique offers the opportunity to take quantitative, three-dimensional measurements of the "run and tumble" behavior exhibited by wild type Escherichia coli $^{28}$ for a population of cells. Until recently, ${ }^{29,30}$ such behavior had been observed in only two dimensions ${ }^{31}$ or in 3D for single cells at a time. ${ }^{32,33}$

Quantitative phase imaging has been recognized as a powerful tool to measure refractive index and dry mass of cells since at least the $1950 \mathrm{~s},{ }^{34,35}$ and its potential to be applied to live biological samples has been acknowledged since the 1990s. ${ }^{36}$ However, the technique had to wait for advances in computational image processing and for lower costs of optomechanical components and lasers for this potential to be realized widely. ${ }^{6}$ Quantitative phase imaging is easily facilitated by digital holographic microscopy and has been used to quantify the shape, volume, movement, membrane tension, phase, and other biophysical parameters of erythrocytes, ${ }^{37-39}$ yeast, ${ }^{40}$ myoblasts, ${ }^{41}$ neural cells, ${ }^{42}$ and trypanosomes. ${ }^{43}$

In this paper, we demonstrate a digital holographic microscope (DHM) capable of operating in inline and offaxis modes, between which the user can conveniently switch. Recording hardware has been optimized for high-speed video acquisition of up to 2000 frames per second (fps). The microscope can be easily configured to record inline holograms at a range of magnifications from $22.5 \times$ to $225 \times$. This mode is suitable for tracking the 3D motion of bacteria and microscopic dielectric particles over a square field of view of side length from $32 \mu \mathrm{m}$ to $320 \mu \mathrm{m}$ and to a depth of $100 \mu \mathrm{m}$. When configured for off-axis operation, the user can choose between bright field and dark field illumination regimes. Dynamic, 
quantitative phase data of biological samples can be taken when the microscope is operated in bright field off-axis mode, allowing for measurements of the refractive index or the surface profile of objects. A novel approach that combines laser dark field microscopy ${ }^{44}$ with holography allows 3D localization of small, yet brightly scattering, objects that cannot be seen ordinarily under bright field conditions. This mode can be used to localize gold nanoparticles, which are ideal tracers for PIV studies of micro-fluid flows ${ }^{45}$ to a depth of at least $16 \mu \mathrm{m}$. In our setup, dark field off-axis holograms were formed at high magnification $(225 \times)$ while bright field off-axis holograms could be acquired at any magnification.

\section{METHODS}

\section{A. Design of multi-mode digital holographic microscope}

\section{Optical design}

A schematic of our multi-mode digital holographic microscope is shown in Fig. 2. It is a custom-built transmission microscope with the off-axis imaging organized around the geometry of a Mach-Zender interferometer. Coherent and linearly polarized light from a diode laser $(635 \mathrm{~nm}, 20 \mathrm{~mW}$; iFLEX-2000, Qioptiq Photonics Ltd), coupled onto a vibration
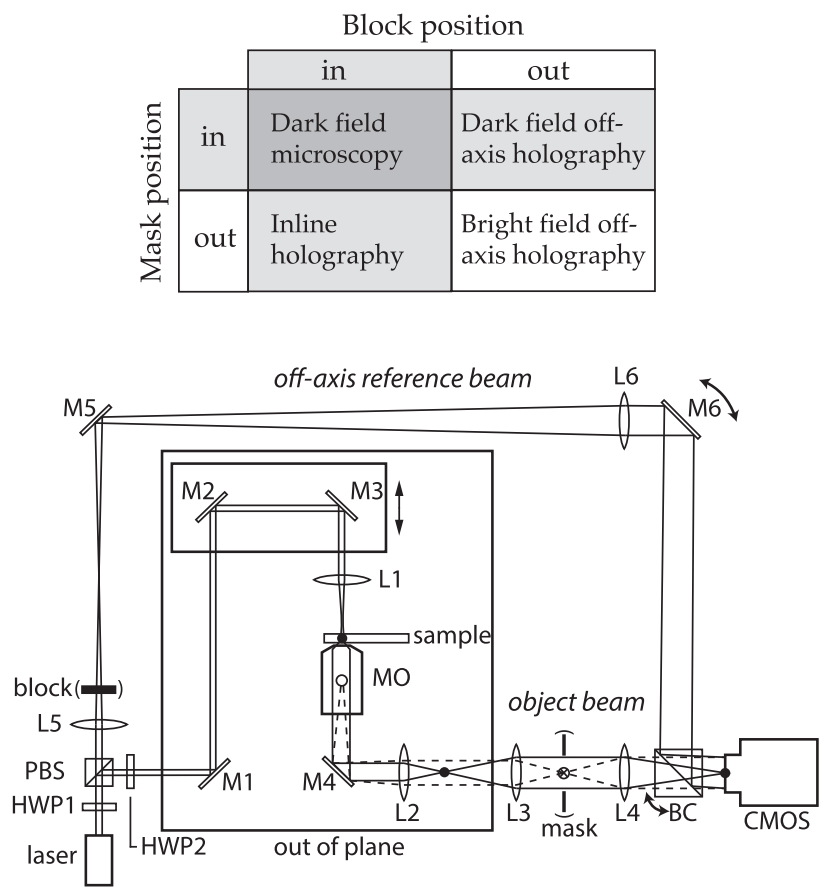

FIG. 2. Schematic of our multi-mode digital holographic microscope. The labels and operation of the microscope are described in the text. The table summarizes the imaging options available to the user depending on the position of the block and mask (indicated in parentheses on the schematic) in the optical path. Solid circles and lines in the object beam indicate conjugate sample planes and rays originating at a point in the sample, respectively. Open circles and dashed lines indicate conjugate back focal planes and rays originating in the objective back focal plane, respectively. isolation optical table via a single-mode fiber, is split into two beam paths using a polarized beam splitter (PBS). One beam path is coupled vertically via mirror M1 to illuminate the sample. For operation in the inline mode, this is the only beam path employed (a movable block serves to occlude the second beam path) and the illumination doubles as the reference beam. This beam is focused to a diffraction-limited spot by lens L1 at the sample plane. The illuminated field of view depends on the focal length of L1 and is thus adjustable.

The sample is introduced through microscope "tunnel slides," whereby a tunnel is formed by sandwiching a slide and cover glass over two strips of double-sided tape or two lines of vacuum grease applied using a syringe. Fine control of the sample is achieved by an xyz piezoelectric nanopositioning stage (P-611.3S NanoCube with E-664 LVPZT amplifier and servo controller; Physik Instrumente $\mathrm{GmbH}$ ). The light scattered off the sample is termed the object beam.

A microscope objective (MO) collects the light from the sample; lenses L2-L4 increase magnification and focus the light to form an image on a complementary metal-oxide semiconductor (CMOS) sensor [1.3 Megapixel (Mpx), $14 \mu \mathrm{m}$ square pixels; EoSens CL, Mikrotron $\mathrm{GmbH}$ ]. This arrangement of lenses also creates a conjugate back focal plane between L3 and L4. For inline holography, Plan-Fluor microscope objectives (Nikon Instruments Inc.) ranging from $10 \times$ to $100 \times$ were used. With lenses L2-L4, this results in a total magnification range of $22.5 \times$ to $225 \times$.

\section{Off-axis operation}

For operation in the off-axis mode, the block is removed and the second beam proceeds through a telescope formed by lenses L5 and L6, which magnifies it to the dimensions of the CMOS sensor. This arm is the off-axis reference beam and interferes with the object beam at a non-polarizing, 50:50 beam recombiner cube (BC) immediately before the camera. Both the mirror M6 and the beam recombiner are adjustable, allowing the angle of interference between the reference and object beams to be controlled. The relative intensity of the two beams is adjusted by the half-wave plate (HWP1) immediately before the polarizing beam splitter. A second half-wave plate (HWP2) in the object beam path is adjusted to ensure the polarization of the two beams is collinear when they interfere. The coherence length of the laser is sub-millimeter, selected to minimize the effects of parasitic scatterers on optical elements in inline imaging. To ensure maximum interference in the off-axis mode, mirrors M2 and M3 are placed on a movable stage with a screw micrometer control to adjust the path difference between the two optical arms.

For dark field off-axis imaging, a physical mask consisting of a chrome steel ball bearing ( $0.5 \mathrm{~mm}$ diameter; Simply Bearings Ltd) mounted on a crosshair is inserted into the conjugate back focal plane between lenses L3 and L4. This has the effect of blocking the undiffracted illumination beam and lowest spatial frequencies of the object beam, forming a dark field image of the sample, which then interferes with the reference beam to create the hologram. This technique results in an increased contrast between the object and reference beams, allowing for samples that are difficult to observe under conventional 
bright field microscopy to be imaged holographically. Lenses L2 and L3 are located to form a conjugate back focal plane at the mask for a particular $100 \times$ microscope objective (PlanFluor, oil immersion; Nikon Instruments, Inc.). The mask is easily removable allowing the return to bright field imaging and could easily be moved to match size and position of the back focal plane of other objectives.

If desired, the microscope can be configured for nonholographic, laser dark field imaging by inserting both the block and the mask. The imaging combinations are summarized in the table in Fig. 2.

\section{Image recording hardware}

The Mikrotron EoSens camera can record full-frame images $(1280 \mathrm{px} \times 1024 \mathrm{px})$ at up to $500 \mathrm{fps}$ and $512 \mathrm{px} \times 512 \mathrm{px}$ subregions (used in this work) at up to $2000 \mathrm{fps}$. Image data are passed to a computer via a microEnable IV framegrabber (VD4-CL; Silicon Software GmbH) and written to a solid-state hard drive (OCZ IBIS 240 GB; OCZ Technology Group, Inc.). This configuration of hardware facilitates the recording of 8-bit 512 px $\times 512$ px images at 2000 fps with no buffering delays until the hard drive fills up. Custom software was written to optimize camera control and to minimize dropped frames and write delays.

\section{B. Holographic reconstruction procedure}

\section{Recorded hologram}

The recorded intensity of a hologram $\left(I_{h}\right)$ is the superposition of the reference $\left(\tilde{R}_{h}\right)$ and object $\left(\tilde{O}_{h}\right)$ terms (see Fig. 1). Its general form is

$I_{h}=\left|\tilde{R}_{h}+\tilde{O}_{h}\right|^{2}=\left|\tilde{R}_{h}\right|^{2}+\left|\tilde{O}_{h}\right|^{2}+\tilde{R}_{h} \tilde{O}_{h}^{*} \mathrm{e}^{-i \mathbf{k} \cdot \mathbf{r} \sin \theta}+\tilde{R}_{h}^{*} \tilde{O}_{h} \mathrm{e}^{+i \mathbf{k} \cdot \mathbf{r} \sin \theta}$,

where $\mathbf{k}$ is the wavevector of the illumination source, $\mathbf{r}$ is a general position vector, and $\theta$ is the angle offset between reference and object beams ( $=0$ for an inline geometry). ${ }^{*}$ signifies the complex conjugate.

\section{Isolating the object term}

The first step in digital holographic reconstruction is to isolate numerically the real object term from both the reference beam and the virtual object term in the recorded hologram intensity.

With inline holography, in order to preserve the complex electromagnetic field of the reconstruction, it is necessary to record multiple holograms per time point, with a phase shift introduced between each hologram. ${ }^{13}$ Instead, to preserve the temporal resolution, we follow the approach taken by Lee and Grier. ${ }^{46}$ A good approximation for the first term in Eq. (1) can be acquired by recording a separate hologram of the reference beam. For motile samples, we typically use a median image of the experimental time series. The $\left|\tilde{R}_{h}^{2}\right|$ term is subtracted from and then used to divide $I_{h}$. We then make the assumptions that the reference beam is a plane wave $\left(\tilde{R}^{*}=\tilde{R}\right)$, that any change in phase between the object and reference beams is negligible, and that the intensity of the reference beam is much greater than that of the object beam, thus $|\tilde{R}|^{2} \gg|\tilde{O}|^{2}$. This leads
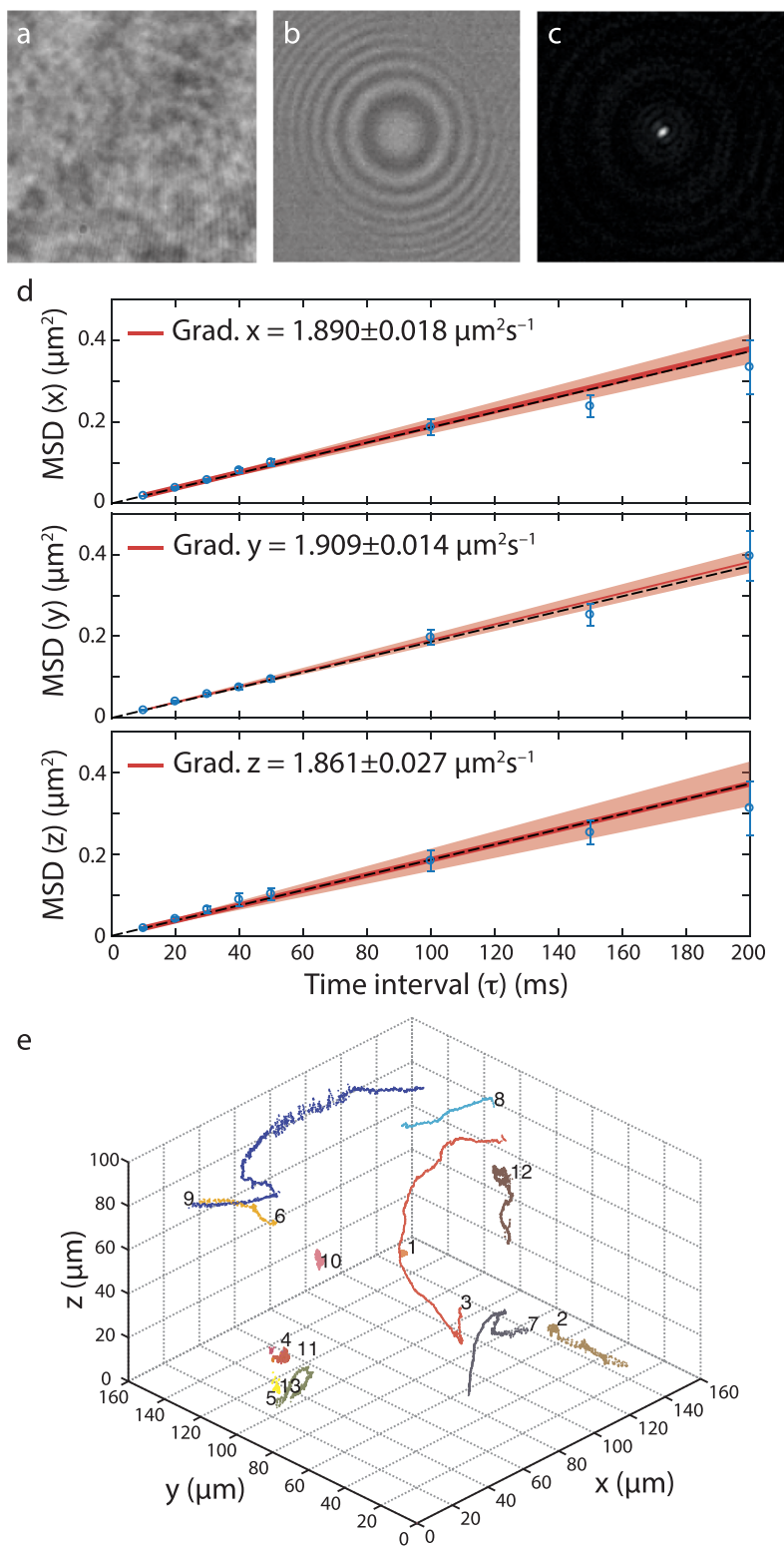

FIG. 3. Three-dimensional micro-particle tracking with inline holography. In [(a)-(c) and (e)], the sample is a dilute suspension of wild type $E$. coli in a tape tunnel slide recorded at $45 \times$ magnification. A $25 \mu \mathrm{m}$ field around a single bacterium is shown in $[(\mathrm{a})-(\mathrm{c})]$; the full field of view can be seen in Fig. S1. The raw hologram (a) is normalized as described in Sec. II B 2 to generate (b). The diffraction pattern caused by light scattered off the bacterium is now clear. The radial size of this pattern corresponds to the distance of the object from the focal plane. A reconstruction $60.8 \mu \mathrm{m}$ above the hologram plane is shown in (c): the bacterium is now in focus. (d) Shows mean square displacement (MSD) of $0.5 \mu \mathrm{m}$ polystyrene microspheres versus elapsed time (blue data points with standard error indicated) with linear fits (red solid line). The gradient of the fits is indicated in the legend; the uncertainty corresponds to the $95 \%$ confidence interval of the fit, also shown in the red shading. The dashed black line is the theoretical value derived from the Stokes-Einstein equation (see Sec. III B). For one dimension, the diffusion coefficient is $0.5 \times$ the gradient value. (e) Reveals the behavior of motile wild type $E$. coli recorded over $40 \mathrm{~s}$. The numbers indicate the start of each trace. "Run and tumble" behavior is clearly visible. 

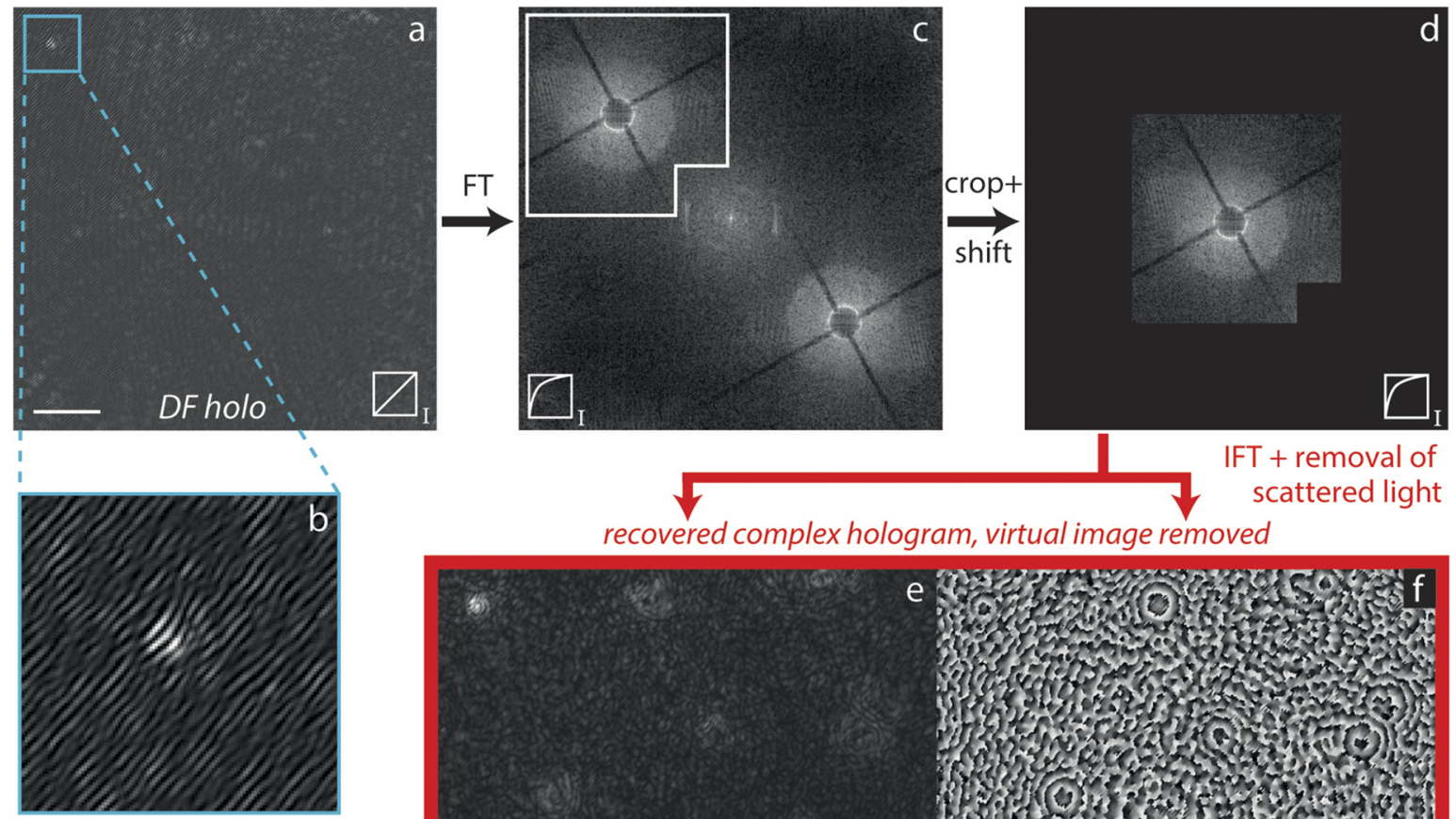

recovered complex hologram, virtual image removed
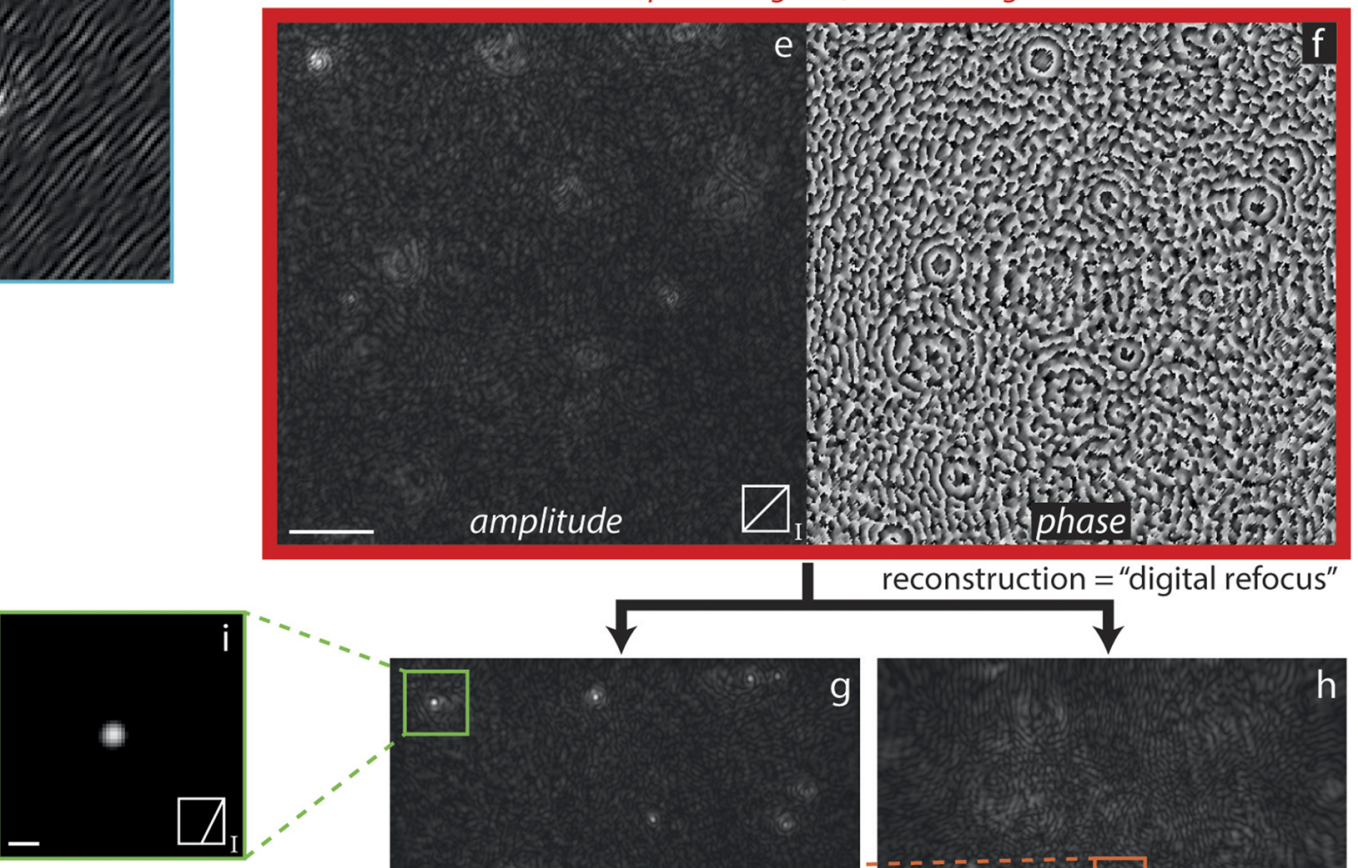

reconstruction $=$ "digital refocus"
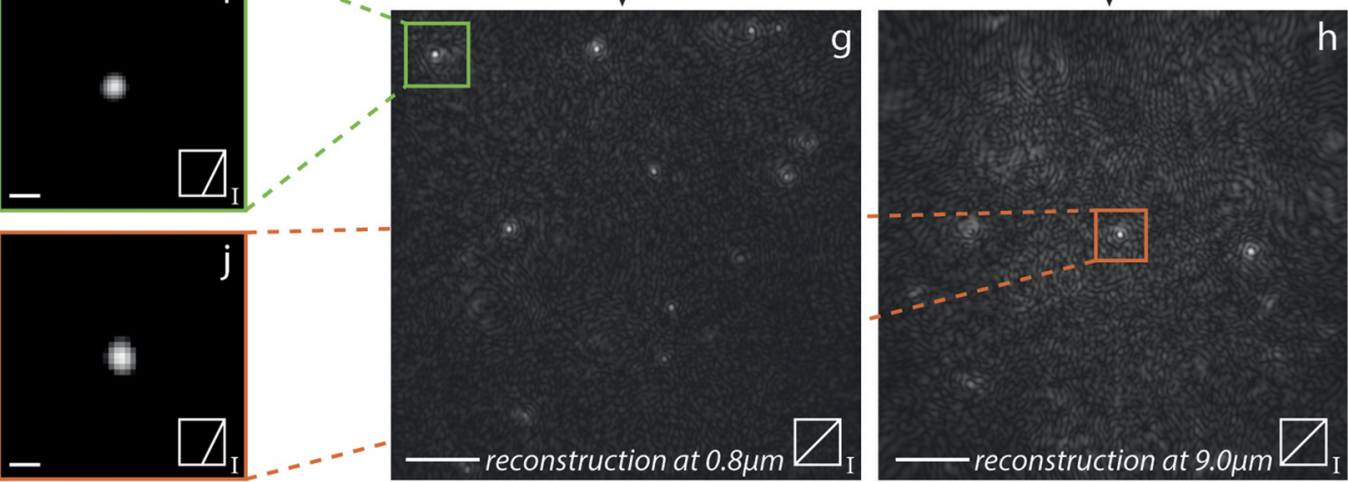

FIG. 4. Localization of gold nanoparticles in 3D using dark field off-axis holography. (a) Shows the intensity of a dark field (DF) hologram of a field of $100 \mathrm{~nm}$ gold nanoparticles. A zoom around one nanoparticle close to the focal plane is shown in (b). The contrast of (b) has been enhanced to show that the signal from the nanoparticle manifests itself as a modulation of the characteristic striped interference pattern of the object and reference beams. To isolate the object term, first a Fourier transform (FT) of the dark field hologram is taken (c). The ball bearing mask and its crosshair supports are visible in the Fourier transform, which also shows light scattered around the edge of the mask. Spatial frequencies corresponding to the real image are cropped and shifted to the center of the Fourier plane (d); a corner of the selected spatial frequencies is clipped to avoid residual low spatial frequency ( $\left(\tilde{O}^{2}{ }^{2}\right.$ terms) before an inverse Fourier transform (IFT) gives both amplitude (e) and phase (f) images. The signal from the light scattered by the mask is removed by background subtraction after the IFT (see S4). From the recovered complex object field [(e) and (f)], the sample can be reconstructed at a user-defined distance from the hologram plane. (g) and $(\mathrm{h})$ show amplitude reconstructions at $0.8 \mu \mathrm{m}$ and $9.0 \mu \mathrm{m}$, respectively. In each case, the nanoparticle shown in the box is in focus. The zoomed images (i) and (j) have had a threshold applied to isolate the gold nanoparticles from the background. This procedure is used to identify the positions in three-dimensional space of the nanoparticles. The axes show the scale used to display the images: image intensity (I) is along the horizontal axis, and gray level $(0-255)$ is along the vertical axis. Scale bars are $5 \mu \mathrm{m}[(\mathrm{a}),(\mathrm{e}),(\mathrm{g})$, and $(\mathrm{h})]$ and $500 \mathrm{~nm}[(\mathrm{i})$ and $(\mathrm{j})]$. 
to an expression for the real component of the object beam expressed entirely in recorded intensities,

$$
\mathbb{R}\left\{\tilde{O}_{h}\right\} \approx \frac{1}{2} \frac{I_{h}}{\left|\tilde{R}_{h}\right|} .
$$

While this method does not allow for a full reconstruction of the complex electromagnetic field of the object beam, it is sufficient for our purposes of localizing the center of a microscopic dielectric object in a 3D volume. Figures 3(a) and 3(b) and S1(a) and S1(b) show the effect of normalizing a raw recorded inline hologram using this approach.

In off-axis holography, the angle $(\theta)$ introduced between the reference and object beams results in a linear phase shift of the real and virtual object terms. This separates the spatial frequencies of these terms from one another and from the $|\tilde{R}|^{2}$ and $|\tilde{O}|^{2}$ terms. Through a numerical operation in Fourier space, the spatial frequencies of the real object term can be identified and isolated, resulting in a complex image of the recorded object beam $\left(\tilde{R}_{h}^{*} \tilde{O}_{h}\right)$. This operation is demonstrated in Figs. 4 and S4 and covered in detail in various texts. ${ }^{10,12,47}$

The multiplicative reference term is eliminated through division by the square root of a separate recording of the intensity of reference beam, which is assumed to be a plane wave, leaving the complex object term isolated.

\section{Holographic reconstruction}

We use the Rayleigh-Sommerfeld formalism ${ }^{48}$ to reconstruct the 3D object field from the isolated hologram object term. This term is convolved with the propagator,

$$
\tilde{\mathrm{H}}(\mathbf{r}, z)=-\frac{1}{2 \pi} \frac{\partial}{\partial z} \frac{\mathrm{e}^{i k \varrho}}{\varrho}
$$

with $\varrho=\sqrt{\left(\mathbf{r}^{2}+z^{2}\right)}$ being the distance from the hologram to the reconstructed object $\left(\tilde{O}_{r}\right)$ along the optical axis $\hat{\mathbf{z}}$, to backpropagate the electromagnetic field at a user-defined distance $d$ along $\hat{\mathbf{z}}$

$$
\tilde{\mathrm{O}}_{r}(\mathbf{r}, d)=\tilde{\mathrm{O}}_{h}(\mathbf{r}, 0) * \tilde{\mathrm{H}}(\mathbf{r}, d) .
$$

A range of $d$ values is set by the user and reconstruction proceeds iteratively, resulting in a volume of electromagnetic data, which is interrogated by subsequent operations (see Sec. III A). These operations are carried out using bespoke software written in Python. The reconstruction operation is highly parallelizable; for this reason, graphics processing units (GPUs) (Tesla C2070, 448 CUDA cores, $5376 \mathrm{MB}$ memory; NVIDIA Corporation) are employed for computational efficiency. For a 512 px $\times 512$ px hologram, our system achieves processing times of $15 \mathrm{~ms} /$ slice to reconstruct, apply an intensity-weighted mask, and write to disk the centers of any objects found. Spacing between reconstructed slices is typically set to the value of the effective pixel spacing of the magnified image. Figure 3(c) shows a 2D slice of the reconstruction of a typical inline hologram $60.8 \mu \mathrm{m}$ above the hologram plane, and Figs. 4(g) and 4(h) shows 2D reconstruction slices from a dark field off-axis hologram. Further examples are available in the supplementary material (Figs. S1-S3 and S5).

\section{MICROSCOPIC OBJECT TRACKING IN 4D}

\section{A. Object localization}

In each frame of a typical dataset of microscopic objects in a fluid medium, the position of each object is determined from the holographic reconstruction as follows. First, an intensity threshold is set based on user evaluation of the dataset and a mask applied to the 3D data array. This principle is demonstrated in Figs. 4(i) and 4(j). Contiguous volumes in the $3 \mathrm{D}$ electromagnetic field above this threshold are candidates for objects of interest. Objects are localized by fitting centroids to the masked data, and the 3D coordinates of their centers are saved. Images of objects far out of focus have lower intensity; thus, it may be necessary to adjust the mask threshold relative to the distance from the hologram plane.

Interference effects from the diffraction patterns of neighboring particles can lead to false particle identification. This is mitigated by filtering identified object candidates by volume.

\section{B. Isotropic diffusion of microspheres}

The performance of the microscope was assessed by determining the 3D diffusion coefficient of $0.5 \mu \mathrm{m}$ diameter polystyrene microspheres (07307; Polybead) using the microscope in inline mode at $225 \times$ magnification. Microspheres were introduced to a tape tunnel slide at a density of $2.9 \times 10^{7} \mathrm{ml}^{-1}$. Ten 2000-frame videos were recorded at $2000 \mathrm{fps}$. The 3D positions of one to three microspheres in each video were tracked using inline holographic reconstruction. A total of 18 tracks were recovered. Mean squared displacements (MSDs) were calculated for non-overlapping time intervals $(\tau)$ between $10 \mathrm{~ms}$ and $200 \mathrm{~ms}$ (68 independent intervals for $200 \mathrm{~ms} ; 1676$ for $10 \mathrm{~ms}$ ). A weighted least squares model was used to provide a linear fit to the data, from which the following diffusion coefficients $(D=M S D / 2 \tau)$ were calculated: $\mathrm{D}_{x}=0.945 \pm 0.009 \mu \mathrm{m}^{2} \mathrm{~s}^{-1}, \mathrm{D}_{y}=0.955 \pm 0.007 \mu \mathrm{m}^{2} \mathrm{~s}^{-1}$, and $\mathrm{D}_{z}=0.931 \pm 0.014 \mu \mathrm{m}^{2} \mathrm{~s}^{-1}$. The small deviations from the Stokes-Einstein equation, $\mathrm{D}=k_{\mathrm{B}} \mathrm{T} / 6 \pi \eta r=0.930 \mu \mathrm{m}^{2} \mathrm{~s}^{-1}$, where $k_{\mathrm{B}}$ is Boltzmann's constant, T is the temperature $(296 \mathrm{~K})$, $\eta$ is the viscosity of water at $296 \mathrm{~K}\left(9.321 \times 10^{-4} \mathrm{~Pa} \mathrm{~s}\right)$, and $r$ the is radius of the microsphere, might be due to noise processes other than the Brownian motion of the beads. The results are summarized in Fig. 3(d).

\section{Biological samples}

Motile bacteria were also tracked in three dimensions using inline holography. Wild type Escherichia coli (strain RP43749) was used for these experiments. In the motile phase, this strain naturally exhibits chemotactic "run and tumble" swimming behavior. Cell cultures were incubated at $30^{\circ} \mathrm{C}$ on an orbital agitator platform at $140 \mathrm{rpm}$ in $5 \mathrm{ml}$ T-broth (1\% Bacto Difco tryptone and $0.5 \% \mathrm{NaCl}$ ) until the motile phase was reached $\left(\mathrm{OD}_{600 \mathrm{~nm}} \simeq 0.6\right.$; typically after $\sim 6 \mathrm{~h}$ incubation). Cells were then washed in motility buffer as described ${ }^{50}$ and diluted to the desired concentration (calibrated experimentally to ensure $10-50$ bacteria in a microscope field of view). 
Motile cells were introduced to a tape tunnel slide. With a magnification setting of $45 \times, 2000$-frame sets of holograms were recorded at $50 \mathrm{fps}$ and the $3 \mathrm{D}$ positions of the cells in each frame output during holographic reconstruction. The reconstructed volume is $160 \mu \mathrm{m} \times 160 \mu \mathrm{m} \times 100 \mu \mathrm{m}$. The tracks of cells are plotted in $3 \mathrm{D}$ to give a qualitative display of cell motility [Fig. 3(e)]. The "run and tumble" behavior of motile wild type cells is clear; non-motile cells are also evident from traces that exhibit diffusion due to Brownian motion. The supplementary material shows a series of holographic reconstructions from the frame displayed in Fig. 3(c) at different heights above the sample plane (Fig. S2) along with a video demonstrating a scroll through this reconstructed volume at $0.5 \mu \mathrm{m}$ steps from 0 to $100 \mu \mathrm{m}$ above the sample plane (Fig. S3).

We anticipate this mode of the microscope could be used for high-throughput quantitative studies of bacterial motility. Straightforward adjustments to the magnification could see it employed for larger micro-organisms also.

\section{APPLICATIONS OF OFF-AXIS HOLOGRAPHIC MICROSCOPY}

\section{A. Localization of gold nanoparticles}

Gold nanoparticles are of interest as tracer particles in PIV studies of the microflows in microfluidic devices ${ }^{51}$ and around swimming micro-organisms such as bacteria; 52 however, high-contrast microscopy techniques, such as dark field back-scattering, ${ }^{44}$ are required to visualize them, which typically work only for in-focus particles. When set to operate in dark field off-axis mode (see Sec. II A 2), our digital holographic microscope is capable of localizing $100 \mathrm{~nm}$ gold nanoparticles (GC100; BB International) at least $16 \mu \mathrm{m}$ from the focal plane.

A ball bearing mounted on a crosshair is inserted in a conjugate back focal plane of the optical train to be used as a mask (see Fig. 2). This mask removes most of the unscattered light from the illumination beam, thus allowing for greater sensitivity in recording weakly scattering objects. An equivalent sensitivity is not possible under bright field illumination due to camera saturation. A small amount of leaked light scatters off the mask, which we remove by subtracting a complex reconstruction of this time-invariant leaked light field from all other frames in the recording. See Fig. S4 for details of this operation.

After removal of the leaked light field, the desired object term is isolated from the dark field interference pattern via an operation in Fourier space (see Sec. II B 2), the 3D light field reconstructed (see Sec. II B 3), and nanoparticles are localized in 3D. Figure 4 summarizes this procedure.

The sample is a solution of $100 \mathrm{~nm}$ gold nanoparticles, at number density $1.87 \times 10^{9} \mathrm{ml}^{-1}$, introduced to a grease tunnel slide with the two glass surfaces separated by a $50 \mu \mathrm{m}$ shim. Fields of view of $30 \mu \mathrm{m} \times 30 \mu \mathrm{m}$ were recorded at $225 \times$ magnification at $100 \mathrm{fps}$. The exposure time of each frame was set to $40 \mu$ s to avoid motion blur in the image. Datasets were recorded within $5 \mathrm{~min}$ of preparation of the sample to minimize sedimentation of nanoparticles to the floor of the chamber.
The technique results in a 3D reconstruction of the complex field of a dark field image. The location of gold nanoparticles is determined via their intensity above a user-defined threshold [Figs. 4(i) and 4(j)], and particles were able to be detected to at least $16 \mu \mathrm{m}$ above the focal plane (see Fig. S5). The phase image of the field reveals ring structures [Fig. 4(f)] indicating the $3 \mathrm{D}$ position of nanoparticles even when they are not visible in the raw hologram. Figure S5 contains more examples of dark field holographic reconstruction.

\section{B. Phase recovery}

Off-axis holographic imaging allows the complex electromagnetic signal emanating from the sample to be recovered. Operating the microscope in bright field off-axis mode thus permits quantitative measurement of the phase of the sample. Phase data of the sample can be used to determine the refractive index of a sample, if its thickness is known, or to generate the 3D surface profile of the imaged object, facilitating topographical studies of biological, and other, samples. To demonstrate this capability, part of a resolution test target (positive 1951 USAF wheel pattern; Thorlabs, Inc.) [Figs. 5(a) and 5(b)] and a dilute suspension of human erythrocytes in isotonic $\mathrm{NaCl}$ [Figs. 5(c)-5(f)] were imaged in bright field off-axis imaging mode at $225 \times$ magnification.

Holograms were acquired with the image plane set to the floor of the sample chamber using the piezoelectric nanopositioner. In each case, a hologram of the background (no object in the field of view, but the same refractive index) of the sample was acquired. The complex electromagnetic field was recovered through holographic reconstruction (see Sec. II B 3). After unwrapping, the phase of the background was subtracted from that of the sample to arrive at a phase difference in radians $(\Delta \phi)$. See Fig. S6 for details. From this phase difference, one can determine either refractive index $\left(n_{\text {sample }}\right)$ or height $(h)$ of the sample via the following relation:

$$
\Delta \phi=\frac{2 \pi}{\lambda}\left(n_{\text {sample }}-n_{\text {medium }}\right) h,
$$

where $\lambda$ is the vacuum wavelength of the illumination and $n_{\text {medium }}$ is the refractive index of the medium surrounding the sample.

We determine the mean phase difference between the background and the numeral " 3 " in Fig. 5 (a) to be $1.73 \pm 0.47$ rad. Figure $5(\mathrm{~b})$ shows a representative line scan through the sample. The calculation of the mean phase difference is arrived at by averaging the phase values throughout the background area and the region bounded by the numeral " 3 ." The test target is manufactured by sputtering chromium metal onto soda lime glass; the thickness of the deposition is given as $120 \mathrm{~nm} \pm 10 \%$. We thus calculate the mean refractive across the target to be $2.78 \pm 0.80$. Measuring the refractive index of metals is notoriously difficult and typically only performed for films up to several tens of nanometers thick. ${ }^{53,54}$ The refractive index of thin-film chromium ${ }^{55}$ under $635 \mathrm{~nm}$ illumination is given as 3.14 ; whereas that of chromium oxide, ${ }^{53}$ which spontaneously forms on the surface of metallic chromium in air, is 2.5. Thus our result is consistent with a mixture of chromium and its oxide of the specified thickness. 

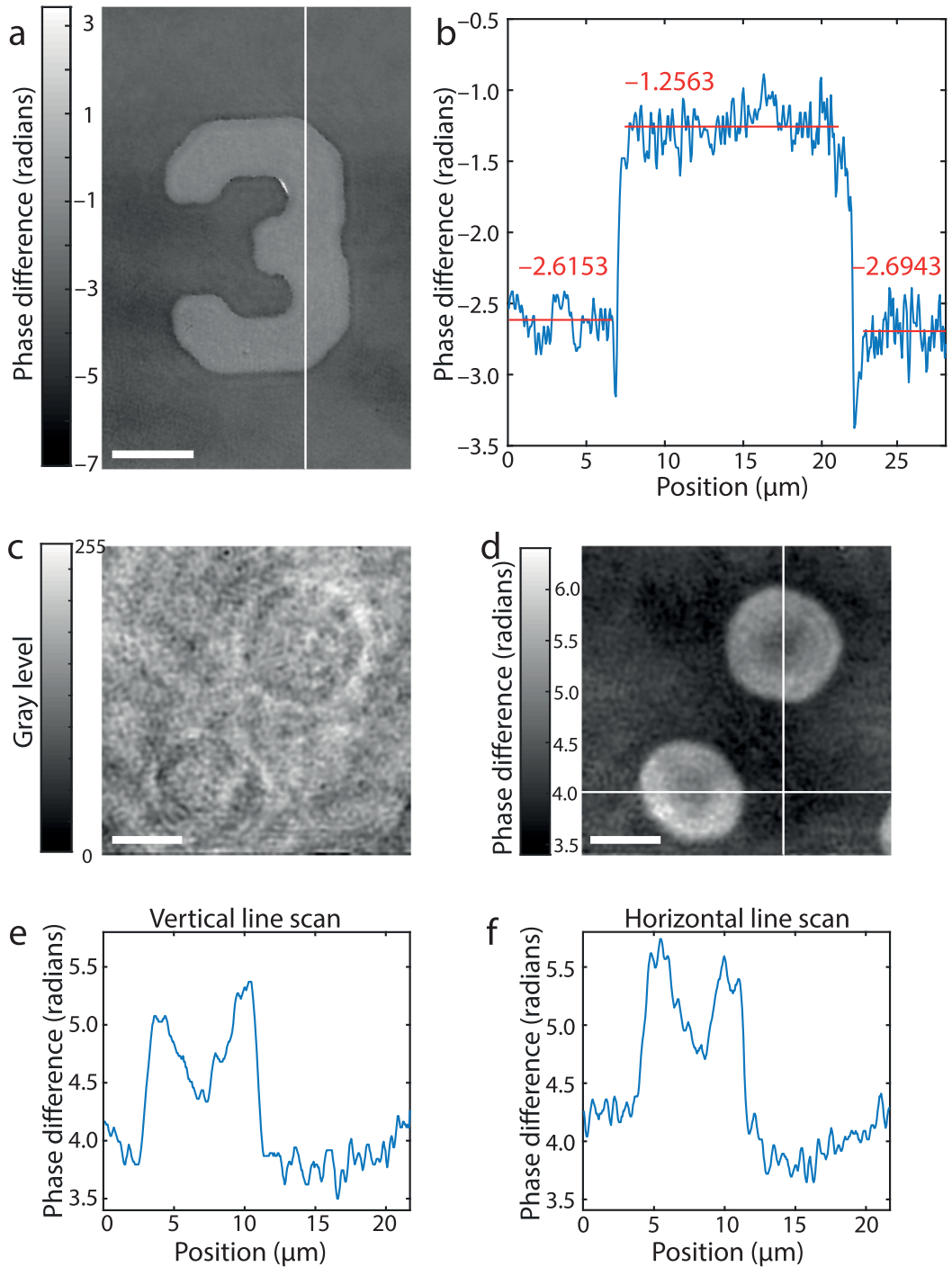

FIG. 5. Recovery of phase information and topographical detail using bright field off-axis holography. A phase difference map of a section of a USAF resolution target is shown in (a). Following reconstruction of the complex electromagnetic field of the target hologram and a background hologram, the difference map is acquired by subtracting the unwrapped phases from one another. The scale bar is $5 \mu \mathrm{m}$. (b) shows the line profile indicated in (a) with numbers indicating the mean value of the horizontal lines fitted to the phase. In $[(\mathrm{c})-(\mathrm{f})]$, the sample is human erythrocytes in saline solution. (c) shows the magnitude image of the hologram, and (d) shows the phase difference map. The scale bars are $5 \mu \mathrm{m}$, and (e) and (f) show the vertical and horizontal line profiles, respectively. Figure S6 details the process of phase unwrapping.
Figures $5(\mathrm{c})-5(\mathrm{f})$ show the power of this technique to reveal quantitative topographical information in samples that are near-invisible under conventional bright field microscopy. The characteristic biconcave disc morphology of erythrocytes is revealed by taking line scans through the centers of the cells under quantitative phase imaging.

The dry mass of biological samples can also be determined. The phase difference between a cell and the surrounding medium is given by $^{34}$

$$
\Delta \phi=2 \pi \frac{d m}{d \mathrm{~A}} \frac{\alpha}{\lambda}
$$

where $d m / d \mathrm{~A}$ is the local mass per unit area and $\alpha$ is an empirically derived constant of proportionality known as the specific refractive increment. For red blood cells, ${ }^{56}$ $\alpha=0.19 \mu \mathrm{m}^{3} \mathrm{pg}^{-1}$.
We calculated the dry mass of the sample cells shown in Figs. 5(c) and 5(d) via

$$
m=\int \frac{\Delta \phi}{2 \pi} \frac{\lambda}{\alpha} d \mathrm{~A},
$$

where the integral was performed over the area of each cell. Our results are $27.1 \pm 8.6 \mathrm{pg}$ (top-right) and $25.5 \pm 9.1 \mathrm{pg}$ (bottom-left), which are consistent with dry mass values found in the literature. ${ }^{57}$

\section{CONCLUSION}

Digital holographic microscopy offers a powerful approach for studying the 3D behavior of microscopic objects in fluid environments such as populations of bacteria and other micro-organisms, and inert particles that could be used as tracers for $3 \mathrm{D}$ microflow studies. The technique also allows 
the phase of a sample to be measured dynamically and, from this information, one can determine the refractive index or topography of the sample.

We have developed a multi-mode digital holographic microscope capable of operating in three distinct holographic imaging modalities, as well as performing conventional laser dark field microscopy. Holograms can be acquired at a range of magnifications from $22.5 \times$ to $225 \times$, and at speeds up to $2000 \mathrm{fps}$, facilitating the study of high-speed 3D swimming dynamics of cells. It is easy to switch between the different imaging options.

Our microscope facilitates the tracking in three dimensions of motile cells, micro-organisms, dielectric microspheres, and gold nanoparticles. The latter is achieved through a novel introduction of a physical mask in a conjugate Fourier imaging plane. In addition, our microscope can measure the phase of biological samples dynamically, allowing biophysical parameters such as refractive index and dry mass to be measured in live samples.

\section{SUPPLEMENTARY MATERIAL}

See supplementary material for further illustrations of holographic processing techniques and more examples of 3D localization of motile bacteria and gold nanoparticles.

\section{ACKNOWLEDGMENTS}

This work was supported by EPSRC Basic Technologies Grant No. EP/F041306/1, BBSRC Strategic Tools and Resources Development Fund Grant No. BB/J020885/1 and the Oxford University John Fell Fund.

\section{REFERENCES}

${ }^{1}$ D. Gabor, Nature 161, 777 (1948).

2J. Sheng, E. Malkiel, and J. Katz, Appl. Opt. 45, 3893 (2006).

${ }^{3}$ P. Marquet, B. Rappaz, P. J. Magistretti, E. Cuche, Y. Emery, T. Colomb, and C. Depeursinge, Opt. Lett. 30, 468 (2005)

${ }^{4}$ N. Warnasooriya, F. Joud, P. Bun, G. Tessier, M. Coppey-Moisan, P. Desbiolles, M. Atlan, M. Abboud, and M. Gross, Opt. Express 18, 3264 (2010).

${ }^{5}$ F. Verpillat, F. Joud, P. Desbiolles, and M. Gross, Opt. Express 19, 26044 (2011).

${ }^{6}$ P. Marquet, C. Depeursinge, and P. J. Magistretti, Neurophotonics 1, 020901 (2014).

${ }^{7}$ D. B. Kirk and W. M. W. Hwu, Programming Massively Parallel Processors: A Hands-on Approach, 3rd ed. (Elsevier, Cambridge, MA, 2016).

${ }^{8}$ S.-H. Lee, Y. Roichman, G.-R. Yi, S.-H. Kim, S.-M. Yang, A. van Blaaderen, P. van Oostrum, and D. G. Grier, Opt. Express 15, 18275 (2007).

${ }^{9}$ L. Wilson and R. Zhang, Opt. Express 20, 16735 (2012).

${ }^{10}$ U. Schnars and W. Jüptner, Digital Holography: Digital Hologram Recording, Numerical Reconstruction, and Related Techniques (Springer-Verlag, Berlin, Heidelberg, 2005).

${ }^{11}$ E. N. Leith and J. Upatnieks, J. Opt. Soc. Am. 52, 1123 (1962).

${ }^{12}$ E. Cuche, C. Depeursinge, and P. Marquet, Appl. Opt. 39, 4070 (2000).

${ }^{13} \mathrm{~T}$. Zhang and I. Yamaguchi, Opt. Lett. 23, 1221 (1998).

${ }^{14}$ T. Tahara, K. Ito, T. Kakue, M. Fujii, Y. Shimozato, Y. Awatsuji, K. Nishio, S. Ura, T. Kubota, and O. Matoba, Biomed. Opt. Express 1, 610 (2010).

${ }^{15}$ X. Lu, J. Chen, S. Liu, Z. Ma, Z. Zhang, and L. Zhong, Opt. Lasers Eng. 50, 1431 (2012).

${ }^{16} \mathrm{~W}$. Xu, M. H. Jericho, I. A. Meinertzhagen, and H. J. Kreuzer, Appl. Opt. 41, 5367 (2002)
${ }^{17}$ R. J. Adrian, Exp. Fluids 39, 159 (2005).

${ }^{18}$ J. S. Guasto, K. A. Johnson, and J. P. Gollub, Phys. Rev. Lett. 105, 168102 (2010).

${ }^{19}$ S. T. Wereley and C. D. Meinhart, Annu, Rev. Fluid Mech. 42, 557 (2010).

${ }^{20}$ Z. Frentz, S. Kuehn, D. Hekstra, and S. Leibler, Rev. Sci. Instrum. 81, 084301 (2010).

${ }^{21}$ E. Malkiel, J. Sheng, J. Katz, and J. R. Strickler, J. Exp. Biol. 206, 3657 (2003).

${ }^{22}$ B. J. Gemmell, J. Sheng, and E. J. Buskey, Proc. Natl. Acad. Sci. U. S. A. 110 , 4661 (2013).

${ }^{23}$ J. Sheng, E. Malkiel, J. Katz, J. Adolf, R. Belas, and A. R. Place, Proc. Natl. Acad. Sci. U. S. A. 104, 17512 (2007)

${ }^{24}$ T. W. Su, L. Xue, and A. Ozcan, Proc. Natl. Acad. Sci. U. S. A. 109, 16018 (2012).

${ }^{25}$ D. F. Blair, Annu. Rev. Microbiol. 49, 489 (1995).

${ }^{26}$ H. C. Berg, Annu. Rev. Biochem. 72, 19 (2003).

${ }^{27}$ Y. Sowa and R. M. Berry, Q. Rev. Biophys. 41, 103 (2008).

${ }^{28}$ G. H. Wadhams and J. P. Armitage, Nat. Rev. Mol. Cell Biol, 5, 1024 (2004).

${ }^{29}$ F. C. Cheong, C. C. Wong, Y. Gao, M. H. Nai, Y. Cui, S. Park, L. J. Kenney, and C. T. Lim, Biophys. J. 108, 1248 (2015).

${ }^{30}$ A. Wang, R. F. Garmann, and V. N. Manoharan, Opt. Express 24, 23719 (2016).

${ }^{31}$ J. C. Conrad, M. L. Gibiansky, F. Jin, V. D. Gordon, D. A. Motto, M. A. Mathewson, W. G. Stopka, D. C. Zelasko, J. D. Shrout, and G. C. L. Wong, Biophys. J. 100, 1608 (2011).

${ }^{32}$ H. C. Berg, Rev. Sci. Instrum. 42, 868 (1971).

${ }^{33}$ L. Turner, L. Ping, M. Neubauer, and H. C. Berg, Biophys. J. 111, 630 (2016).

${ }^{34}$ R. Barer, Nature 169, 366 (1952).

${ }^{35}$ R. Barer, J. Opt. Soc. Am. 47, 545 (1957).

${ }^{36}$ G. A. Dunn, Proc. Royal Microsc. Soc. 33, 189 (1998)

${ }^{37}$ G. Popescu, T. Ikeda, K. Goda, C. A. Best-Popescu, M. Laposata, S. Manley, R. R. Dasari, K. Badizadegan, and M. S. Feld, Phys. Rev. Lett. 97, 218101 (2006). ${ }^{38}$ P. Girshovitz and N. T. Shaked, Opt. Express 21, 5701 (2013).

${ }^{39}$ N. Cardenas and S. K. Mohanty, Appl. Phys. Lett. 103, 013703 (2013).

${ }^{40}$ B. Rappaz, E. Cano, T. Colomb, J. Kühn, C. Depeursinge, V. Simanis, P. J. Magistretti, and P. Marquet, J. Biomed. Opt. 14, 034049 (2009).

${ }^{41}$ M. F. Toy, C. Pache, J. Parent, J. Kühn, M. Egli, and C. Depeursinge, Proc. SPIE 7570, 75700A (2010).

${ }^{42}$ P. Marquet, C. Depeursinge, and P. J. Magistretti, Annu. Rev. Biomed. Eng. 15, 407 (2013).

${ }^{43}$ G. G. Romero, A. C. Monaldi, and E. E. Alanís, Opt. Commun. 285, 1613 (2012).

${ }^{44}$ Y. Sowa, B. C. Steel, and R. M. Berry, Rev. Sci. Instrum. 81, 113704 (2010).

${ }^{45}$ S. I. Satake, N. Unno, S. Nakata, and J. Taniguchi, Meas. Sci. Technol. 27, 084009 (2016)

${ }^{46}$ S.-H. Lee and D. G. Grier, Opt. Express 15, 1505 (2007).

${ }^{47} \mathrm{~V}$. Toal, Introduction to Holography (CRC Press, Boca Raton, 2012).

${ }^{48} \mathrm{~J}$. W. Goodman, Introduction to Fourier Optics, 3rd ed. (Roberts \& Company, Englewood, 2005)

${ }^{49}$ J. S. Parkinson, J. Bacteriol. 135, 45 (1978).

${ }^{50}$ J. Adler and M. M. Dahl, Microbiology 46, 161 (1967)

${ }^{51}$ M. Dienerowitz, M. Lee, G. Gibson, and M. Padgett, Lab Chip 13, 2359 (2013).

${ }^{52}$ S. S. Haque, "Applications of nanoparticle image velocimetry in nanofluids," M.Sc. thesis, University of Tennessee, 2011.

${ }^{53}$ R. M. Hill and C. Weaver, Trans. Faraday Soc. 54, 1140 (1958).

${ }^{54}$ F. L. McCrackin, E. Passaglia, R. R. Stromberg, and H. L. Steinberg, J. Res. Natl. Inst. Stand. Technol. 106, 589 (2001).

${ }^{55}$ P. B. Johnson and R. W. Christy, Phys. Rev. B 9, 5056 (1974).

${ }^{56}$ S. Aknoun, J. Savatier, P. Bon, F. Galland, L. Abdeladim, B. Wattellier, and S. Monneret, J. Biomed. Opt. 20, 126009 (2015).

${ }^{57}$ K. G. Phillips, S. L. Jacques, and O. J. T. McCarty, Phys. Rev. Lett. 109, 118105 (2012). 